\title{
Laparotomy for Acute Intestinal Obstruction in a Tertiary Care Center
}

\author{
${ }^{1}$ Kodenge R Rao, ${ }^{2}$ Samir R Nayak, ${ }^{3}$ Dileep Sunkavalli, ${ }^{4}$ Reshma Palacharla, ${ }^{5}$ Kurra V Devi
}

\begin{abstract}
Background: Acute intestinal obstruction is one of the most common causes of surgical admissions worldwide. Adhesions ${ }^{1}$ appear to be the most common cause in the Western world as well as in parts of Asia and the Middle East. But in India, it has been observed that obstructed inguinal hernia seemed to be the most prevalent cause. Radiography accurately diagnoses intestinal obstruction in approximately $60 \%$ of cases. The etiological factors and diagnostic approach seems to vary in the different demographic study. In this study, an attempt has been made to elaborate on our experience in acute intestinal obstruction with its cause and management.
\end{abstract}

Aim: To discuss the surgical management in regard to 40 cases of acute intestinal obstruction.

Methods: A retrospective analysis of 40 cases that underwent exploratory laparotomy for an indication of acute intestinal obstruction in the period between June 2016 and May 2018.

Results: Of the 40 cases that were included in the study has been observed that the median age of patients has been 51.4 years and $16(40 \%)$ of cases were due to adhesions, $12(30 \%)$ cases were due to malignant growths which comprised the major etiological causes. The mortality rate was $15 \%$, and the complication rate was $25 \%$. Accurate diagnosis on radiography could only be obtained in $4(10 \%)$ cases.

Conclusion: The analysis of patient data reveals that postoperative adhesions is a major etiological factor for acute intestinal obstruction in India similar to the western world and that CT abdomen must be opted as a means for accurate diagnosis prior to surgical approach.

Keywords: Acute intestinal obstruction, Adhesion, Laparotomy.

How to cite this article: Rao KR, Nayak SR, Sunkavalli D, Palacharla R, Devi KV. Laparotomy for Acute Intestinal Obstruction in a Tertiary Care Centre. J Med Sci 2018;4(4): 103-106.

\section{Source of support: Nil}

Conflict of interest: None

\section{INTRODUCTION}

Small bowel obstruction is the leading cause of emergency hospital admission worldwide. ${ }^{1,2}$

\footnotetext{
${ }^{1,2}$ Professor, ${ }^{3-5}$ Resident,

1-5Department of Surgery, GSL Medical College, Rajahmundry, Andhra Pradesh, India
}

Corresponding Author: Samir R Nayak, Professor, Department of Surgery, GSL Medical College, Rajahmundry, Andhra Pradesh, India, e-mail: Drsamirnayak@Gmail.com
There has been some progress in our knowledge of the pathological physiology and treatment of acute intestinal obstruction in the past two decades. Despite this advance, the mortality rate in the treatment of such obstructions remains discouragingly high. There are differences of opinion among persons with considerable experience as to certain aspects of management, but this does not seem to be importantly related to the continued high mortality rate.

In the developed world approximately $20 \%$ of patients with acute abdominal pain admitted to surgical units have an intestinal obstruction, and $80 \%$ of these will have a small bowel obstruction. Malignancy is the leading cause of obstruction of the large intestine. The cardinal clinical features are vomiting, abdominal pain, distension, and gross constipation which differ in predominance depending on the site of the obstruction. ${ }^{3}$ Radiography in form of X-ray abdomen and CT abdomen accurately diagnose the cause of bowel obstruction in approx $60-70 \%$ of cases. ${ }^{4}$ Management of bowel obstruction requires prompt identification, meticulous attention to fluid and electrolyte balance and timely surgical intervention. Failure of early diagnosis and delayed application of proved sound therauptic procedure undoubtlyare the most important factors in the maintainace of ltethal results of acute abstruction. ${ }^{5}$

\section{METHODS}

This case study comprises a retrospective analysis of 40 cases that underwent emergency laparotomy for acute intestinal obstruction in the period between June 2016 and May 2018. There was no age limit to the cases selected. All cases underwent investigations as seen fit and were handled by a team comprising of a consultant level surgeon, anesthetist, and radiologist. Initial resuscitation and fluid management were carried out in all cases. An X-ray erect abdomen and computed tomography (CT) scan were performed in all patients in whom intestinal obstruction was suspected. All patients were managed for a time period seen fit in an intensive care unit (ICU) after the procedure and were evaluated over a 30-day postoperative period or discharge, whichever was earliest and were thus categorized into three patient outcome groups as recovered $(\mathrm{R})$, recovered with complication (RC) and death (D). 


\section{RESULTS}

\section{Age and Sex Determination}

Acute intestinal obstruction was seen to affect males (22 patients, 55\%) than females (18 patients, $45 \%$ ) almost equally (Table 1) and adhesions which were the most common etiology (16 cases, $40 \%$ ) affected males and females at a ratio of 1:1. The most common occurrence was in the age group $40-70$ years (22 patients, $55 \%$ ), while the least common occurrence was in the age group $<20$ years (1 patient, $2.5 \%$ ). The mean age of our patients was 41.27 years while the median age was 51.44 years.

\section{Etiology}

The etiological factors have been enumerated.

The most common presenting complaint for which these patients received consultation and were admitted was distention in $35(87.5 \%)$ cases, obstipation in $34(85 \%)$ cases, pain in $31(77.5 \%)$ cases, vomiting in $29(72.5 \%)$ cases, mass felt per abdomen in 5 (12.5\%) cases. Most of these patients ( 34 patients, $85 \%$ ) had presented to the hospital 48 hours after the initial onset of their complaint and a minor amount (6 patients, $15 \%$ ) sought medical care within 48 hours of their complaint (Graph 1).

Criteria for subjecting patients to surgery included increasing pain and distension with gross abdominal tenderness, tachycardia, and other features of toxicity like fever and leukocytosis and failure of nonoperative treatment. Twelve (30\%) of the cases that underwent operative management for intestinal obstruction were found to have features of peritonitis on admission. Seven (17.5\%) cases were found to have leukocytosis upon admission.

\section{Investigation}

X-ray abdomen erect and CT abdomen was performed in all patients in whom acute intestinal obstruction was suspected before deciding on a conservative or surgical approach. It was observed that decision making was unaltered by CT regarding surgical approach. In only $4(10 \%)$ cases whereas the decision to go for a trial of conservative management was abandoned after $\mathrm{CT}$ in the remaining $36(90 \%)$ cases.

Table 1: Age distribution amongst various patients operated for acute intestinal obstruction

\begin{tabular}{lll}
\hline Age Range & Number & Percentage \\
\hline $10-20$ & 1 & 2.5 \\
$20-30$ & 5 & 12.5 \\
$30-40$ & 4 & 10 \\
$40-50$ & 8 & 20 \\
$50-60$ & 7 & 17.5 \\
$60-70$ & 7 & 17.5 \\
$70-80$ & 2 & 5 \\
\hline
\end{tabular}

\section{Management}

Prompt diagnosis and decision making allowing early management within 24 hours of arrival were possible in $30(75 \%)$ cases whereas the decision took an average time of 2.6 days in the remaining patients. Appropriate surgical intervention was performed often involving a multidisciplinary approach especially in cases where malignancy was suspected. The patients were then managed in the surgical ICU during the postoperative period. Postoperative ventilator support was required due to inability to extubate the patient in $5(12.5 \%)$ cases.

\section{Complications}

Complications were seen in $10(25 \%)$ cases. The most common complication was related to a stoma in $4(10 \%)$ of cases. Respiratory infection in $2(5 \%)$ cases, wound infection in $1(2.5 \%)$ case, anastomotic leak in $1(2.5 \%)$ case, burst abdomen in $1(2.5 \%)$, and relaparotomy in $1(2.5 \%)$ case.

\section{Mortality}

It was seen that $6(15 \%)$ patients expired after surgical intervention and of these $3(50 \%)$ cases were due to superior mesenteric ischemia which carried a 100\% mortality rate amongst the cases dealt with in our institution.

\section{DISCUSSION}

It has been observed that the incidence of acute intestinal obstruction has been similar in both males and females. Adhesions proved to be the most common etiological factor despite the belief that obstructed hernia is to be the most common cause for such an event in the Indian subcontinent. Souvik et al. cited in their article obstructed hernia is the main cause of intestinal obstruction followed by malignancy and adhesion but the number of cases of obstructed hernia encountered during our study period

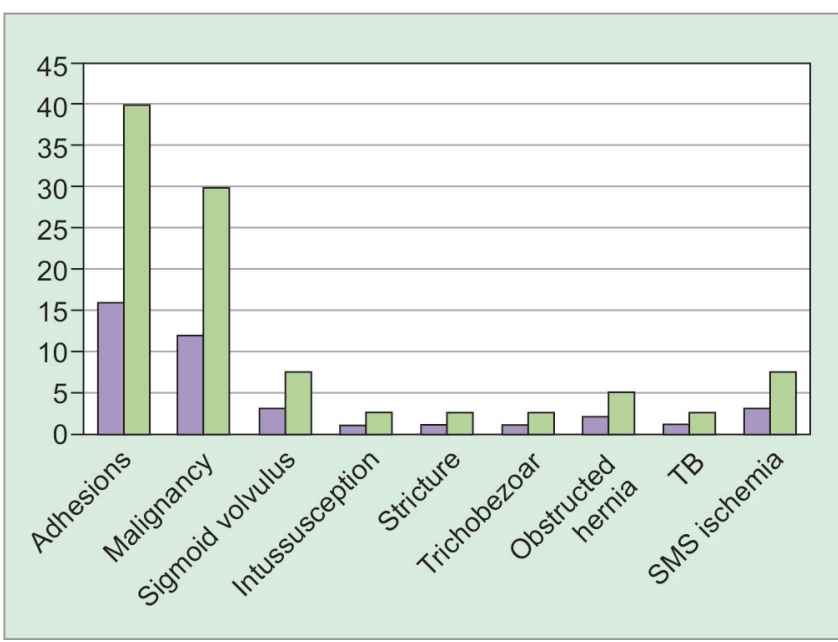

Graph 1: Etiologies causing intestinal obstruction that resulted in surgical intervention 
has been trivial compared to the number of cases caused due to postoperative adhesions and malignancy. ${ }^{2}$

The feeling of a distended abdomen appears to be the most frequently cited complaint on presentation, and a majority of these cases are found to seek medical care well after 48 hours of the appearance of these symptoms. The absence of passage of flatus and/or feces and abdominal distensions are the most common symptoms and physical finding of patients with acute mechanical bowel obstruction, respectively. ${ }^{3-5}$

This appears to be a halfway meet between the awareness of acute abdominal conditions, outreach of medical facilities and the impending ignorance amongst the general population. Patients with superior mesenteric artery ischemia belonged to the age group of above 60 years and presented with a complaint of pain that was out of proportion to findings on examination. ${ }^{6,7}$ Features of peritonitis could be elicited in 12 (30\%) of the cases and could not prove an accurate predictor of patient outcome.

In contrast to the historical diagnostic accuracy of X-ray abdomen erect in diagnosis and management of acute intestinal obstruction it has been our experience that a CT abdomen could unmask conditions that needed urgent surgical intervention which might have otherwise been treated conservatively had the approach been confined to basing judgment upon radiography alone. ${ }^{8}$ This was of peculiar importance in case of superior mesenteric artery ischemia wherein all 3 cases dealt with in this healthcare facility it has been noted that the initial radiograph upon admission was noted to be remarkably normal. In our first experience, the patient was managed conservatively upon this finding and a similarly normal $X$-ray at 24 hours after admission only to leave us with a bitter misdiagnosis and a fatality. 3,9,10

In cases involving adhesions adhesiolysis was performed, and viability of the bowel was inspected upon which gangrenous changes had ensued in a segment of the small bowel in 8 (50\%) of the 16 cases where resection and anastomosis was performed. Two (12.5\%) of these cases expired and $1(6.25 \%)$ case recovered with complications.

In all 12 cases of malignancy, a diversion stoma was made with or without resection of the malignant lesion depending upon the need for further assessment and approach. 4 (33\%) of these cases recovered with complications that were related to stoma and $1(8.3 \%)$ case expired after the procedure. Ferguson et al. in their article conclude that if surgery is to be undertaken, resection and anastomosis should be performed in preference to the formation of a stoma or enteric bypass. ${ }^{11}$ Ultimately, the aim of any therapy in intestinal obstruction is to restore intestinal function, but if this fails for whatever reason, management should enable the patient to be cared for in their chosen environment, which may not be an acute hospital. In our study, all case attend emergency with terminally ill and some cases are not fit for prolonging surgery due to associated anemia and malnutrition and hence palliative stoma procedure was obtained with prior consent.

In all three cases of sigmoid volvulus, sigmoidectomy was performed with primary anastomosis using 3-0' Vicryl. Two $(66.7 \%)$ cases recovered without consequence and 1 $(33.3 \%)$ cases recovered with a complication of respiratory tract infection in the postoperative period. The standard surgical practice has been explored, with resection of the sigmoid colon. But given the age and comorbidities of the affected population, resection has been considered highly risky. That said, with advances in anesthesia and critical care technology, resection has become more feasible and safe. ${ }^{12}$

All three cases of superior mesenteric artery ischemia proved to be fatal in our experience with delayed presentation, ambiguous clinical findings and unsalvageable intraoperative findings paired with a deteriorated general condition of the patient and prevalent comorbid heart diseases have proved a lethal combination. It has been difficult to preserve more than $50 \mathrm{~cm}$ of the bowel in any of these cases, and a 100\% mortality has been recorded. The incidence and mortality of AOSMA are higher than previously reported from clinical series. There is seldom any suspicion of the diagnosis prior to death. ${ }^{13}$

In the two cases of obstructed hernia dealt with in our facility inguinal approach was undertaken in both cases with an extension of dissection to involve the posterior wall of the inguinal canal. Viable bowel was encountered in one case where simple reduction and hernioplasty was sufficient whereas gangrenous bowel was noted in the other case which required resection and anastomosis with herniorrhaphy due to unfavorable anatomy and a high chance for infection in case of placement of mesh.

One case of ileocecal intussusceptions was treated which had gangrenous bowel on laparotomy and was dealt with in the form of right hemicolectomy. ${ }^{1}$ case of stricture of the small bowel was operated upon where the stricture was noted in the terminal ileum and resulted in resection and primary anastomosis with 3-0' Vicryl.

One case of trichobezoar was treated by enterotomy and removal of the obstructive pathology followed by primary closure. A trichobezoar is a bezoar made up of hair and is a rare cause of bowel obstruction of the proximal gastrointestinal tract. They are seen mostly in young women with trichotillomania and trichotillophagia. ${ }^{14}$ In our study series, we encountered one case of trichobezoar and treated with laparoscopic enterotomy and repair.

The complication rate in the postoperative management was around $25 \%$, and the mortality was $15 \%$ in our study group. Major factors influencing the rates were age, comorbidity, nonviable strangulation, and treatment delay. Nonviable bowel and strangulation were more common in old patients. 
The overall complication rate in the various study was $19.8-23 \%$, and the most common complications in that study were wound infections and peritonitis. ${ }^{15}$ In our study, the major complications were cardiovascular and pulmonary ones; this may be due to old age followed by a high frequency of comorbidity.

\section{CONCLUSION}

Adhesions prove to be the most significant cause of acute intestinal obstruction in this region of India closely followed by malignant obstruction with a remarkably low amount of cases due to obstructed inguinal hernia or intestinal tuberculosis. SMA ischemia is a medical challenge associated with near total mortality. CECT abdomen proves to be a powerful tool and not just an adjunct in timely and accurate diagnosis and operative decision making.

\section{REFERENCES}

1. Moran BJ. Adhesion-related small bowel obstruction. Colorectal Dis. 2007 Oct;9(Suppl 2):39-44.

2. Souvik A, Hossein MZ, Amitabha D, Nilanjan M, Udipta R. Etiology and outcome of acute intestinal obstruction: A review of 367 patients in Eastern India. Saudi J Gastroenterol. 2010 Oct;16(4): 285-287.

3. Markogiannakis H, Messaris E, Dardamanis D, Pararas N, Tzertzemelis D, Giannopoulos P, et al. Acute mechanical bowel obstruction: clinical presentation, etiology, management and outcome. World J Gastroenterol. 2007 Jan 21;13(3):432-437.

4. Maglinte DD, Heitkamp DE, Howard TJ, Kelvin FM, Lappas JC. Current concepts in imaging of small bowel obstruction. Radiologic Clinics. 2003 Mar 1;41(2):263-283.
5. Coller FA. Acute obstruction of the small bowel. JAMA: The Journal of the American Medical Association [Internet]. American Medical Association (AMA); 1949 May 14;140(2):135.

6. Bala M, Kashuk J, Moore EE, Kluger Y, Biffl W, Gomes CA, et al. Acute mesenteric ischemia: guidelines of the World Society of Emergency Surgery. World Journal of Emergency Surgery. 2017 Dec;12(1):38.

7. Stephenson JA, Singh B. Intestinal obstruction. Surgery (Oxford) [Internet]. Elsevier BV; 2011 Jan;29(1):33-38.

8. Jackson PG, Raiji M. Evaluation and management of intestinal obstruction. American family physician. 2011 Jan 15;83(2):159-165.

9. Furukawa A, Kanasaki S, Kono N, Wakamiya M, Tanaka T, Takahashi M, Murata K. CT diagnosis of acute mesenteric ischemia from various causes. American Journal of Roentgenology. 2009 Feb;192(2):408-416.

10. Kassahun WT, Schulz T, Richter O, Hauss J. Unchanged high mortality rates from acute occlusive intestinal ischemia: six year review. Langenbeck's Archives of Surgery. 2008 Mar 1;393(2):163-171.

11. Ferguson HJ, Ferguson CI, Speakman J, Ismail T. Management of intestinal obstruction in advanced malignancy. Annals of Medicine and Surgery. 2015 Sep 1;4(3):264-270.

12. Gingold D, Murrell Z. Management of colonic volvulus. Clinics in Colon and Rectal Surgery. 2012 Dec;25(4):236-244.

13. Acosta S, Ögren M, Sternby NH, Bergqvist D, Björck M. Incidence of acute thrombo-embolic occlusion of the superior mesenteric artery-a population-based study. European Journal of Vascular and Endovascular Surgery. 2004 Feb 1;27(2):145-150.

14. Gaujoux S, Bach G, Au J, Godiris-Petit G, Munoz-Bongrand N, Cattan P, Sarfati E. Trichobezoar: A rare cause of bowel obstruction. World Journal of Gastrointestinal Surgery. 2011 Apr;3(4):54-55.

15. Fevang BT, Fevang J, Stangeland L, SøreideO, Svanes K, Viste A. Complications and death after surgical treatment of small bowel obstruction: a 35-year institutional experience. Annals of Surgery. 2000 Apr;231(4):529-537. 